\section{Effect of high-fat mixed lipid diet and swimming on fibre types in skeletal muscles of rats with colon tumours}

\author{
Vika Smerdu, ${ }^{1}$ Martina Perše ${ }^{2}$ \\ ${ }^{1}$ Institute of Anatomy, Faculty of \\ Medicine, University of Ljubljana \\ ${ }^{2}$ Institute of Pathology, Medical \\ Experimental Centre, Faculty of \\ Medicine, University of Ljubljana, \\ Slovenia
}

\section{Abstract}

Skeletal muscle fibre types, whose characteristics are determined by myosin heavy chain (MyHC) isoforms, can adapt to changed physiological demands with changed $\mathrm{MyHC}$ isoform expression resulting in the fibre type transitions. The endurance training is known to induce fastto-slow transitions and has beneficial effect in carcinogenesis, whereas the effect of an excessive fat intake and its interaction with the effect of swimming are less conclusive. Therefore, we studied the effect of high-fat mixed lipid (HFML) diet and long-term (21-week) swimming on fibre type transitions and their average diameters by immunohistochemical demonstration of $\mathrm{MyHC}$ isoforms in slow soleus (SOL), fast extensor digitorum longus (EDL), and mixed gastrocnemius medialis and lateralis (GM, GL) muscles, divided to deep and superficial portions (GMd, GMs, GLd, GLs), of sedentary and swimming Wistar rats with experimentally (dimethylhydrazine) induced colon tumours and fed either with HFML or low-fat corn oil (LFCO) diet. HFML diet induced only a trend for fast-to-slow transitions in SOL and in the opposite direction in GMd. Swimming triggered significant transitions in unexpected slow-to-fast direction in SOL, whereas in GMs the transitions had tendency to proceed in the expected fast-toslow direction. The average diameters of fibre types were mostly unaffected. Hence, it can be concluded that if present, the effects of HFML diet and swimming on fibre type transitions were counteractive and muscle-specific implying that each muscle possesses its own adaptive range of response to changed physiological conditions.

\section{Introduction}

It is well established that various physi- ological, pathological and environmental factors, including physical activity affect skeletal muscle contractile and metabolic properties which may reflect in the muscle fibre type transitions. ${ }^{1,2}$ Although fibre types are broadly divided into type 1 and type 2 fibres, skeletal muscle fibres are mostly classified according to the myosin heavy chain $(\mathrm{MyHC})$ isoforms, which are one of the most relevant markers of fibre types and their contractile and metabolic characteristics. There are four major $\mathrm{MyHC}$ isoforms expressed in adult rat skeletal muscles, i.e. MyHC-1, $-2 \mathrm{a},-2 \mathrm{x}(2 \mathrm{~d})$ and $2 b{ }^{3}$ MyHC-1 is expressed in slow-twitch type 1 fibres, which are rich in mitochondria, possess high oxidative capacity, are fatigue resistant and are involved in longlasting tension activities. They are numerous in slow, oxidative, red muscles like the rat soleus (SOL). Type 2 fibres are further subdivided into type $2 \mathrm{a}, 2 \mathrm{x}$, and $2 \mathrm{~b}$ according to the expressed MyHC isoform (MyHC-2a, -2x, and -2b). Type 2b fibres are the fastest ones with predominant glycolytic metabolism and fatigue easily. Type $2 \mathrm{a}$ and $2 \mathrm{x}$ fibres have intermediate contractile and metabolic characteristics between those of type 1 and type $2 \mathrm{~b}$ fibres. Type 2 fibres, particularly type $2 b$ are involved in intense and rapid activities of short duration and are abundant in fast, glycolytic, white muscles. An example of a muscle enriched in type 2 fibres is the rat extensor digitorum longus (EDL) ${ }^{4}$ The fibre type transitions are assumed to mostly proceed gradually $(1 \leftrightarrow 1 / 2 \mathrm{a} \leftrightarrow 2 \mathrm{a} \leftrightarrow 2 \mathrm{ax} \leftrightarrow 2 \mathrm{x} \leftrightarrow 2 \mathrm{xb} \leftrightarrow 2 \mathrm{~b}) .^{5}$ However, such muscle fibre type transitions include removal of old proteins and synthesis and replacement with new ones, which are better suited for particular physiological conditions. ${ }^{6}$

Though the effects of several factors, especially the endurance training, on the muscle fibre type composition have been studied well, the effects of tumorigenesis and long-term high fat diet on muscle fibre type transitions have been less conclusive. To our knowledge, our recent study was the first to demonstrate that an experimentally induced tumorigenesis resulted in altered fibre type proportions in a muscle-specific manner. ${ }^{7}$ On the other hand, the studies on the relationship between high fat diet and muscle fibre type composition in rat are scarce, they are mostly evaluated in the context of the obesity ${ }^{8}$ and their results are contradictory, ${ }^{9-11}$ which is not surprising as it was demonstrated that different fat sources have various effect on the fibre type transitions. ${ }^{12}$

In recent years, high fat diet together with sedentary life style has been recognized as an important factor responsible for various diseases, such as obesity, diabetes,
Correspondence: Vika Smerdu, Institute of Anatomy, Faculty of Medicine, University of Ljubljana, Korytkova 2, 1000 Ljubljana, Slovenia.

Tel.: +386.1.5437306 - Fax: +386.1.5437301 E-mail: vika.smerdu@mf.uni-lj.si

Key words: Myosin heavy chain isoforms; rat; skeletal muscles; immunohistochemistry; high-fat diet; swimming.

Contributions: MP conducted the animal experiments, VS analysed skeletal muscles, and both authors participated equally in data analyses and the manuscript preparation with intellectual and editorial contributions. Both authors approved the manuscript.

Conflict of interests: the authors declare no potential conflict of interests.

Funding: The research was supported by the Slovenian Research Agency grant, no. P30043 and P3-0054.

Acknowledgements: the authors thank to Mrs Ksenija Kodra, Mrs. Nataša Pollak, Mrs. Majda Črnak-Maasarani, Mrs. Andreja Vidmar, Mr. Marko Slak and Mr. Milan Števanec for their technical assistance.

Ethical approval: All applicable international, national and/or institutional guidelines for the care and use of animals were followed. All procedures performed in studies involving animals were in accordance with the ethical standards of the institution or practice at which the studies were conducted.

Conference presentation: Part of this paper was presented at the 43rd European Muscle Conference, September 10-14, 2014, Salzburg, Austria.

Received for publication: 22 May 2018. Accepted for publication: 11 July 2018.

This work is licensed under a Creative Commons Attribution-NonCommercial 4.0 International License (CC BY-NC 4.0).

(C) Copyright V. Smerdu and M. Perše, 2018 Licensee PAGEPress, Italy

European Journal of Histochemistry 2018; 62:2945 doi:10.4081/ejh.2018.2945

cardiovascular diseases, including colon cancer which is one of the most common cancers worldwide. ${ }^{13}$ To investigate the underlying mechanisms through which various physiological and environmental factors interact with colon cancer development, various animal models have been used. One of the well-established models of the experimental multistep development of colon carcinogenesis with morphological and histological features similar to those seen in the human sporadic colon carcinogenesis (i.e., dysplastic crypts, adenomas, 
carcinomas) is the carcinogen 1,2 dimethyhydrazine (DMH) model. ${ }^{14-16}$

Nevertheless, the studies on animal models of colon cancer have shown that the composition of the ingested dietary fatty acids has been a more critical risk factor than the total amount of the ingested fat. Further, it was demonstrated that dietary fatty acids affect the lipid content of tissues, lipid peroxidation process, antioxidant status, arachidonic acid metabolism, immune function, cell signalling pathways, cell proliferation and apoptosis. ${ }^{13}$ Nonetheless, the experimental studies in DMH rat model have shown that high fat diet, particularly high-fat mixed lipid (HFML) diet, which reflects the mixed lipid composition of the average Western-style diet and consists of a mixture of saturated, monosaturated and polyunsaturated fatty acids, has been strongly associated not only with an increased incidence of colon cancer but it compromised the antioxidant defence system in various tissues as well, including the skeletal muscle. ${ }^{17-19}$ On the other hand, the endurance swimming reduced the development of colon tumours in DMH-treated rats on both HFML and low fat corn oil (LFCO) diet but affected the antioxidant defence system in the heart, liver and skeletal muscle only in rats on HFML diet. ${ }^{18,19}$ As already stated, we have demonstrated that $\mathrm{DMH}$ induced tumorigenesis affected the fibre type composition of some muscles, i.e. reduced proportion of hybrid fibre types in most of gastrocnemius samples, but no relevant impact on the SOL and EDL fibre type composition. ${ }^{7}$ On the contrary, the effect of an excessive fat intake and its interaction with the effect of swimming on muscle fibre types in rats with colon tumours have not been evaluated yet.

Therefore, the first aim of our study was to examine whether a long-term (21 weeks) HFML diet affected the fibre type composition of skeletal muscles in rats with DMH induced colon tumours. The second aim was to investigate the potential effect of endurance swimming on the fibre type composition of skeletal muscles of LFCO diet fed rats with DMH induced colon tumours. Further, our third aim was to evaluate whether a long-term swimming (21 weeks) had beneficial effect on the fibre type composition of skeletal muscles of HFML diet fed rats with colon tumours. As a musclespecific response to various factors is known $^{2}$ and the muscles are differently involved in the endurance swimming, four different skeletal muscles were analysed, slow SOL, fast EDL and both heads of mixed gastrocnemius muscles. Nonetheless, we believe that the results of our study may contribute to better understanding the complexity of factors involved in the skeletal muscle adaptation under high fat diet, particularly in the context of colon tumorigenesis.

\section{Materials and Methods}

\section{Animals and experimental protocol}

Twenty-four 4-week-old male Wistar (HsdRccHan $^{\mathrm{TM}}$ : WIST) rats (Medical Experimental Centre, Ljubljana, Slovenia) were used in the study. They were housed at $22-23^{\circ} \mathrm{C}(55 \pm 10 \%$ humidity, $12 \mathrm{~h}$ light/dark cycle). The animal experiment was designed to study the interactive effect of HFML diet and swimming on the incidence of DMH induced colon cancer as already reported in our previous studies. ${ }^{18,19}$ Briefly, after 8 week of adaptation period the animals were randomly divided in 4 experimental groups $(n=6)$ according to the experimental treatment: a pair of swimming (S) and a pair of sedentary groups. Each of the paired groups was provided different diet, either low-fat corn oil (LFCO) or high-fat mixed lipid (HFML) diet. The four animal groups were respectively assigned as LFCO, LFCO-S, HFML, and HFML-S. The rats were treated with carcinogen 1,2dimethylhydrazine (DMH) (Fluka Chemie, Switzerland, $20 \mathrm{mg} / \mathrm{kg}$, dissolved in 0.001 M EDTA; pH 6.8), administered subcutaneously once a week for 15 consecutive weeks. The animals were euthanized 6 weeks after last DMH application at age of 33 weeks. The experimental protocol is summarized in Figure 1 and was approved by the National Ethic Committee of the Republic of Slovenia (License No. 34401$61 / 2007 / 7$ ) and conducted in accordance with the European Convention ETS 123. ${ }^{18,19}$

\section{Diet}

At the age of 12 weeks the rats were provided two types of diet, both based on AIN-76A diet (Altromin, Germany) and prepared according to the diets reported by Rao and co-workers: ${ }^{17}$ a low-fat corn oil diet (LFCO) or a high-fat mixed lipid (HFML) diet. The composition of the experimental diet was adjusted so that both diets offered the same amount of calories, proteins, vitamins, minerals and fibres. LFCO diet consisted of $5 \%$ of total lipids (corn oil), rich in polyunsaturated (PUFA) $\omega-6$ fatty acids, whereas HFML diet consisted of $20 \%$ of total mixed lipids, of which $16 \%$ were derived from beef tallow, $10 \%$ from lard, $12 \%$ from butter fat, $30 \%$ from hydrogenated soy bean oil, $5 \%$ from peanut oil and 27\% from corn oil. HFML diet contained $45 \%$ of saturated, $24 \%$ of monosaturated $(\omega-7 / 9)$ and $28 \%$ of PUFA- $\omega-6$ fatty acids. ${ }^{18,19}$

\section{Swimming}

To adapt to water and swimming, the animals of all four groups were gradually introduced to water over an eight-week adaptation period. Then the animals of the two swimming groups started to exercise in dark phase of the day in $100 \mathrm{~cm}$ high plastic container, filled with $60 \mathrm{~cm}$ deep water (30$33^{\circ} \mathrm{C}$ ) for total 21 weeks 5 days a week. In the first week they swam for $15 \mathrm{~min} /$ day, in the second week they swam for $30 \mathrm{~min}$, thereafter swimming was prolonged for 10 min a week. From the ninth week on, the rats were required to swim $90 \mathrm{~min} /$ day until the end of the experiment (Figure 1). Meanwhile the two swimming groups were exercising, the animals of sedentary groups were kept in a plastic cage with $3 \mathrm{~cm}$ of water $\left(30^{\circ} \mathrm{C}\right)$ to exclude potential stress and other confounding effects.

\section{Muscle samples}

After euthanasia at the age of 33 weeks, four skeletal muscles, known to differ in the fibre type composition, were excised from the right hind limb: slow soleus (SOL), fast extensor digitorum longus (EDL), and the heterogeneous gastrocnemius medialis

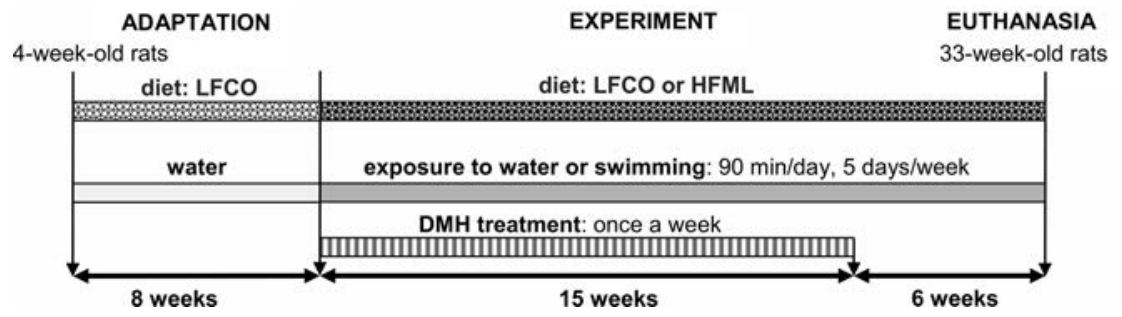

Figure 1. Experimental protocol: low-fat corn oil (LFCO) diet, high-fat mixed lipid (HFML) diet, carcinogen 1,2 dimethylhydrazine (DMH). 
(GM) and lateralis (GL) muscles. Since a prevalence of slower fibre types in the deep part and a predominance of faster ones in the superficial part is characteristic for the gastrocnemius muscles, ${ }^{20} \mathrm{GM}$ and GL samples were further divided into the deep (GMd, GLd) and superficial part (GMs, GLs). The muscle samples were frozen in liquid nitrogen and stored at $-80^{\circ} \mathrm{C}$ until being processed for immunohistochemistry.

\section{Immunohistochemistry}

Fibre type proportions of individual muscles were determined according to the expression of $\mathrm{MyHC}$ isoforms revealed by four monoclonal antibodies: BA-D5 (MyHC-1) and SC-71 (MyHC-2a), 6H1 (MyHC-2x) and BF-F3 (MyHC-2b)..$^{21,22}$ The antibodies BA-D5, SC-71 and BF-F3 were locally produced from cell lines purchased from Deutsche Sammlung von
Mikroorganismen und Zellkulturen (DSMZ, Braunschweig, Germany). The antibody $6 \mathrm{H} 1$ was purchased from the Developmental Studies Hybridoma Bank (DSHB, University of Iowa, USA).

The immunostaining of serial muscle cryosections $(10 \mu \mathrm{m})$ was performed as previously reported. ${ }^{7}$ Briefly, sections were pre-incubated in phosphate buffered saline, containing $0.5 \%$ bovine serum albumin (PBS/BSA) for $30 \mathrm{~min}$. The supernatants with antibodies were appropriately diluted with PBS/BSA (BA-D5 1:500; SC-71 1:200; 6H1 1:50; BF-F3 1:20), the sections with primary antibody were incubated overnight at $4^{\circ} \mathrm{C}$. To verify the specificity of each antibody, a slide parallel to each set of analysed samples was incubated in PBS/BSA lacking the primary antibody. The binding of the antibodies BA-D5, SC71 and BF-F3 was demonstrated using sec- ondary antibody conjugated with horseradish peroxidase (P260, Dako, Glostrup, Denmark), which was diluted $(1: 100)$ in PBS/BSA, with addition of rabbit serum (1:40). To reveal the secondary antibody binding, the sections were incubated in $0.05 \%$ diaminobenzidine tetrahydrocloride hydrate (DAB) and $0.01 \% \mathrm{H}_{2} \mathrm{O}_{2}$ in $0.2 \mathrm{M}$ acetate buffer ( $\mathrm{pH}$ 5.2) for approximately $7 \mathrm{~min}$ in the dark. ${ }^{23,24}$ The binding of 6H1 was demonstrated by NovoLink Polymer Detection System following the instructions of the producer (Leica Biosystems, Newcastle, UK). The control sections were respectively incubated either with the secondary antibody or NovoLink Polymer Detection System.

\section{Muscle sections analysis}

In each of muscle sections, stained with different antibodies, the same selected area
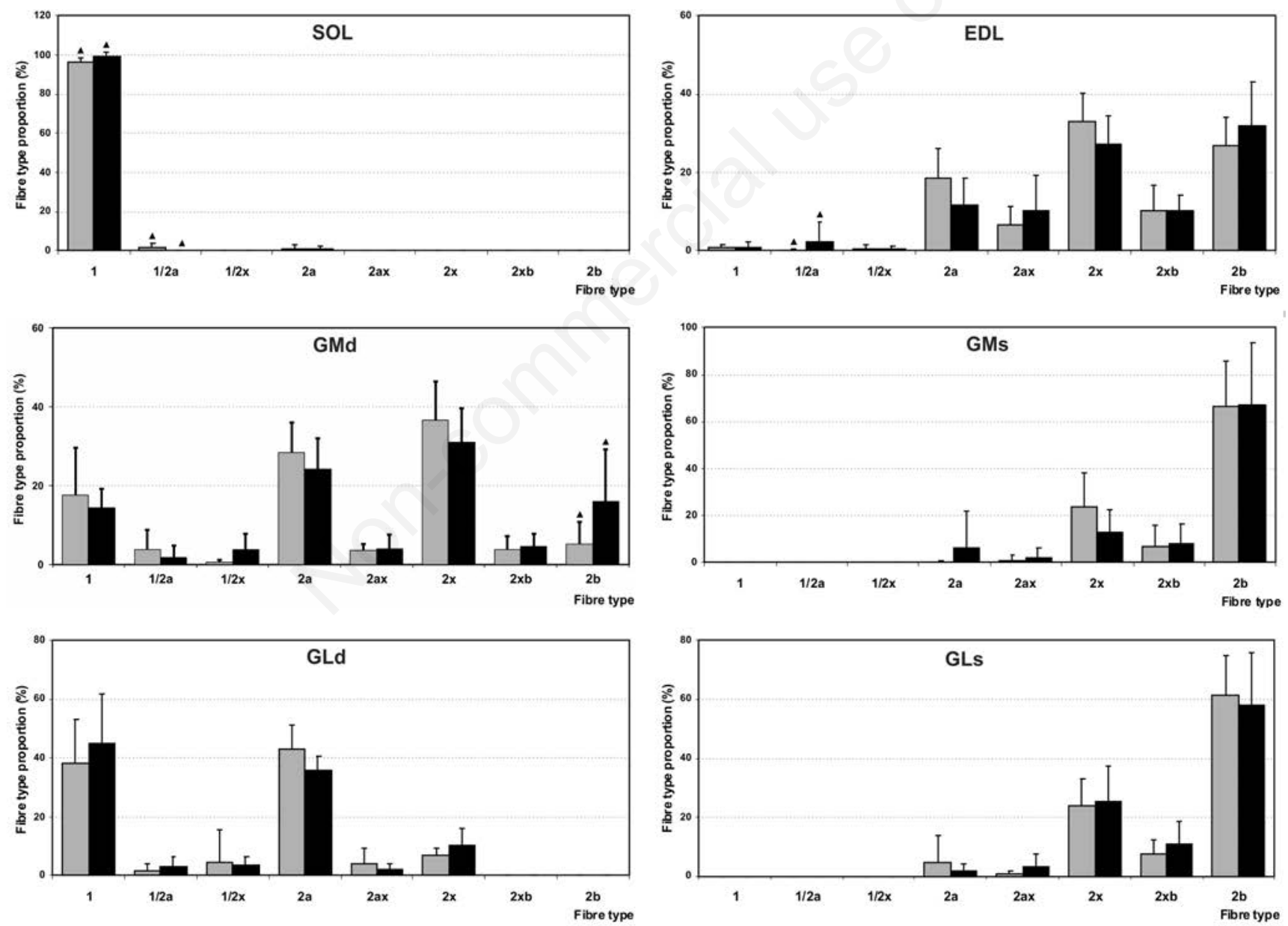

LFCO $\square \mathrm{HFML}$

Figure 2. Effect of high-fat mixed lipid (HFML) diet on fibre type transitions: the average proportions (\%) of fibre types (1, 1/2a, 1/2x, $2 \mathrm{a}, 2 \mathrm{ax}, 2 \mathrm{x}, 2 \mathrm{xb}$ ) in the soleus (SOL), extensor digitorum longus (EDL), the deep and superficial portion of the gastrocnemius medialis (GMd, GMs) and lateralis (GLd, GLs) muscles of low-fat corn oil (LFCO) diet fed sedentary rats (LFCO) and HFML fed sedentary rats $($ HFML). Values are means \pm SEM, significant differences $(\mathrm{P}<0.05)$ between both groups are labelled by an asterisk $(*)$ and the trends for differences $(P=0.05-0.1)$ are labelled by a triangle $(\Delta)$. 
was registered by a digital camera (Nikon, DS-Fi1, 1232 X 972 pixels), connected to a light microscope (Nikon Eclipse 80i, magnification 20x). Thereafter, the registered digital images of serial muscle sections were analysed using a computer-assisted system for image analysis, which was developed in our collaboration with colleagues from the Czech Academy of Sciences. ${ }^{7,25}$ The registered images were subsequently adjusted to fit better to each other using Muscle Reg program. In one of the registered images the contours of approximately 170 fibres were assessed manually using a program Ellipse (ViDiTo, Košice, Slovakia). The set of adjusted images and the superimposed fibre contours were used as input data to computer FibClasM program to determine the labelling pattern of fibres with different antibodies. Finally, using several macros
(Microsoft Excel) fibre types were determined according to their labelling pattern with the four antibodies. In addition, the average diameters of fibre types were computed from the fibre contours.

\section{Statistics}

Statistical analyses were performed using SPSS Statistics program Version 20. The average proportions (\%) and diameters $(\mu \mathrm{m})$ of fibre types were determined within each of the homonymous muscles of the four animal groups using descriptive statistics to calculate means, standard error of the mean (SEM) and the range of all variables. To evaluate an exclusive effect of HFML diet and swimming, the means of LFCO vs HFML and LFCO vs LFCO-S and were compared respectively. To demonstrate the interactive effect of both factors, the values of HFML vs HFML-S were compared.
According to the distribution of the standard deviations of means, determined by a Shapiro-Wilks test, the values of the paired groups were compared either by $t$ test or by Mann-Whitney test. All data reported are means \pm standard error of the mean (SEM). P values below 0.05 were considered significant and those between $0.05-0.1$ as trends for differences.

\section{Results}

\section{Health status of animals}

The body weight of rats was recorded once a week, water and food intake three times per week, clinical state was monitored on daily basis. All animals showed no clinical signs of illness during the entire exper-
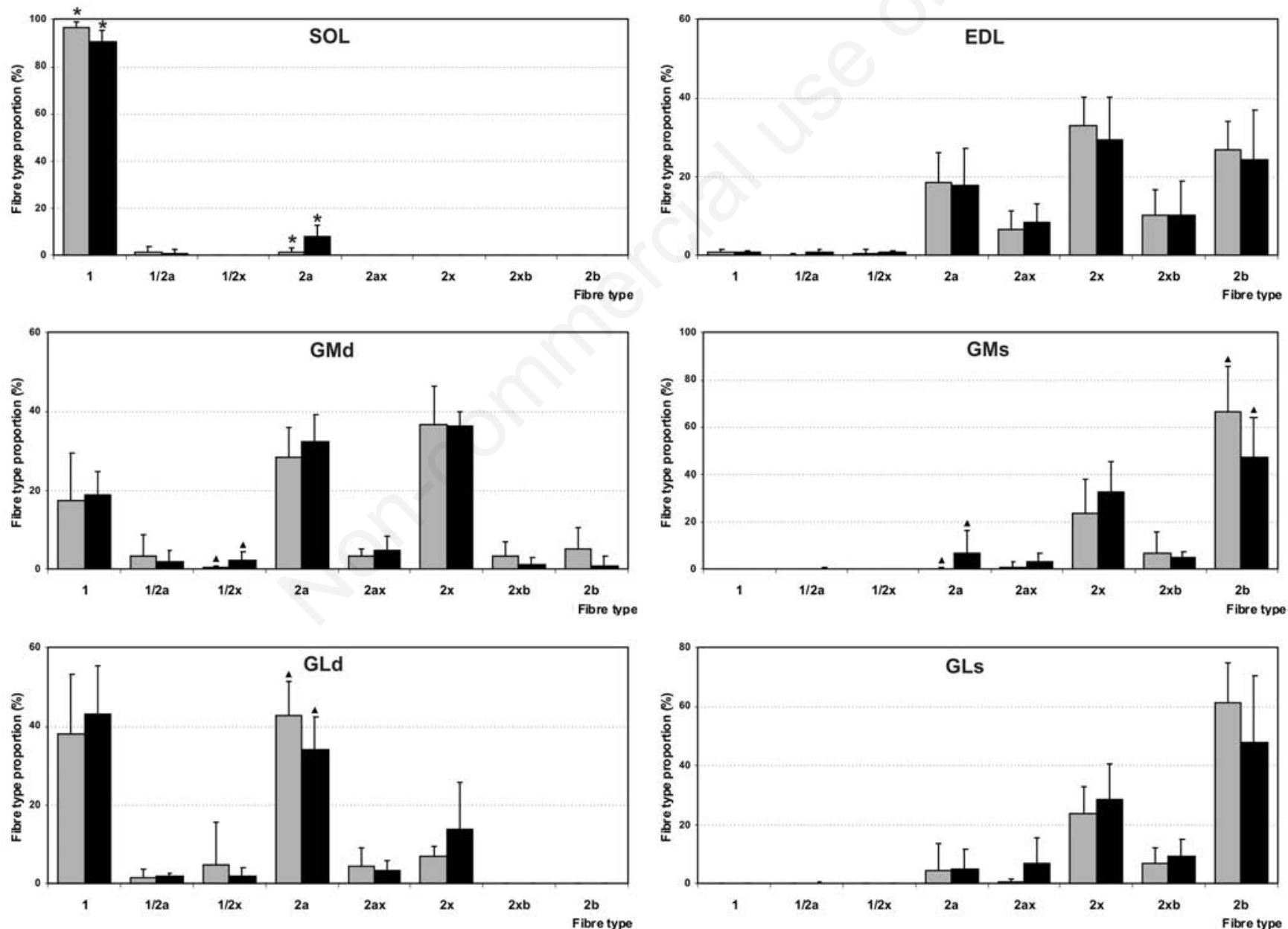

\section{LFCO - LFCO-S}

Figure 3. Effect of swimming on fibre type transitions: the average proportions (\%) of fibre types (1, 1/2a, 1/2x, 2a, 2ax, 2x, 2xb and $2 \mathrm{~b}$ ) in the soleus (SOL), extensor digitorum longus (EDL), the deep and superficial portion of the gastrocnemius medialis (GMd, GMs) and lateralis (GLd, GLs) muscles of low-fat corn oil (LFCO) diet fed sedentary rats (LFCO) and LFCO fed swimming rats (LFCO-S). Values are means \pm SEM, significant differences $(P<0.05)$ between both groups are labelled by an asterisk $(*)$ and the trends for differences $(P=0.05-0.1)$ are labelled by a triangle $(\boldsymbol{\Delta})$. 
iment. The exercising rats consumed significantly higher energy densities than the sedentary rats and the body weights of all rats increased with the advancing age, as demonstrated previously. ${ }^{19}$ The body weight gain of both sedentary and HFML-S rats was comparable during the entire experiment, while the body weight gain of the LFCO-S group became significantly lower from those of the other three groups from the seventeenth week on till the end. At autopsy, a slightly higher but not significant amount of adipose tissue in the abdominal cavity was present in rats of both HFML groups. The pathohistological analyses revealed that the rats developed a small number of colon lesions, mostly macroscopically invisible dysplastic foci of crypts, whereas the adenomas and carcinomas were small and rare..$^{7,18,19}$

\section{Fibre types}

The average proportion of pure fibre types, expressing a single MyHC isoform $(1,2 \mathrm{a}, 2 \mathrm{x}$ or $2 \mathrm{~b})$ and of hybrid fibre types, co-expressing two or even more MyHC isoforms, were determined for each muscle within each animal group. Though 11 different hybrid fibre types could be identified, only the four most numerous ones, i.e. $1 / 2 \mathrm{a}$, $1 / 2 \mathrm{x}, 2 \mathrm{ax}$ and $2 \mathrm{xb}$, were included in the analysis.

\section{Effect of HFML diet}

The effect of HFML diet on fibre types was evaluated by comparison of means of both sedentary groups but fed with different diet, i.e. LFCO vs HFML. HFML diet did not induce any significant differences in the fibre type proportions but only some trends for differences in SOL, GMd and EDL (Figure 2). In SOL a trend of higher type 1 proportion ( $\mathrm{P}=0.093)$ and concomitant type $1 / 2 \mathrm{a}$ absence $(\mathrm{P}=0.065)$ imply transitions towards slow type 1 . Whereas in GMd a trend in the opposite direction, towards faster type $2 \mathrm{~b}$ was evident as their proportion tended to increase $(\mathrm{P}=0.096)$. In EDL there was a trend of type $1 / 2$ a proportion increase $(\mathrm{P}=0.071)$.

\section{Effect of swimming}

To evaluate the effect of swimming on fibre types, the average fibre type proportions of homonymous muscles of sedentary and swimming LFCO groups (LFCO vs LFCO-S) were compared (Figure 3). Swimming induced significant differences only in slow SOL muscle, in which type $2 \mathrm{a}$ proportion significantly increased $(\mathrm{P}=0.007)$ and that of type 1 decreased $(\mathrm{P}=0.033)$, indicating transitions from slow type 1 to faster type $2 \mathrm{a}$ in LFCO-S (Figures 3 and 4 ). But in other muscles there were just trends for differences in the proportions of some fibre types, i.e. an increase of type $1 / 2 \mathrm{x}(\mathrm{P}=0.071)$ in $\mathrm{GMd}$ and a decrease of type $2 \mathrm{a}(\mathrm{P}=0.098)$ proportion in $\mathrm{GLd}$, whereas in GMs lower proportion of type $2 \mathrm{~b} \quad(\mathrm{P}=0.093)$ and higher of type $2 \mathrm{a}$ $(\mathrm{P}=0.065)$ imply a trend for transitions opposite to those in SOL, i.e. from faster type $2 \mathrm{~b}$ towards slower type $2 \mathrm{a}$ fibres.

\section{Interactive effect of HFML diet and swimming}

The interaction of both factors on fibre types was evaluated by comparison of the average fibre type proportions of homonymous muscles of sedentary and swimming HFML groups (HFML vs HFML-S). In SOL a significantly higher proportion of type $1 / 2$ a fibres $(\mathrm{P}=0.015)$ and concomitant trend for lower proportion of type 1 fibres $(\mathrm{P}=0.065)$ imply transitions from slow type 1 towards faster ones (Figure 5). But in GMd, swimming induced transitions in the opposite direction, i.e. from faster fibre types towards slower ones, as the proportions of type $2 \mathrm{xb}(\mathrm{P}=0.023)$ and $2 \mathrm{~b}(\mathrm{P}=0.026)$ fibres were significantly lower. There was also a significantly higher proportion of hybrid type 2 ax fibres $(\mathrm{P}=0.000)$ in $\mathrm{GLd}$, whereas in GMs there was only a trend for higher type $2 \mathrm{x}$ proportion $(\mathrm{P}=0.065)$ in HFML-S.

\section{Effect of HFML diet and swimming on the size of fibre types}

The average diameters of fibre types were mostly unaffected by both HFML diet and swimming (data not shown).

\section{Discussion}

In rats with DMH induced colon tumours we demonstrated that HFML diet and low-intensity, long-term swimming (21 weeks) affected the fibre type composition of SOL muscle and some samples of gastrocnemius muscle, whereas EDL muscle was mostly unaffected. The effects of HFML diet and swimming on fibre type transitions were counteractive and musclespecific, as the transitions induced by each of the two factors proceeded in the opposite direction in SOL and in GMd, whereas the average diameters of fibre types mostly remained unaffected by both factors.

\section{Effect of HFML diet}

In rats with DMH induced colon tumours 21 weeks of HFML diet resulted in no significant changes in the fibre type composition of skeletal muscles. We

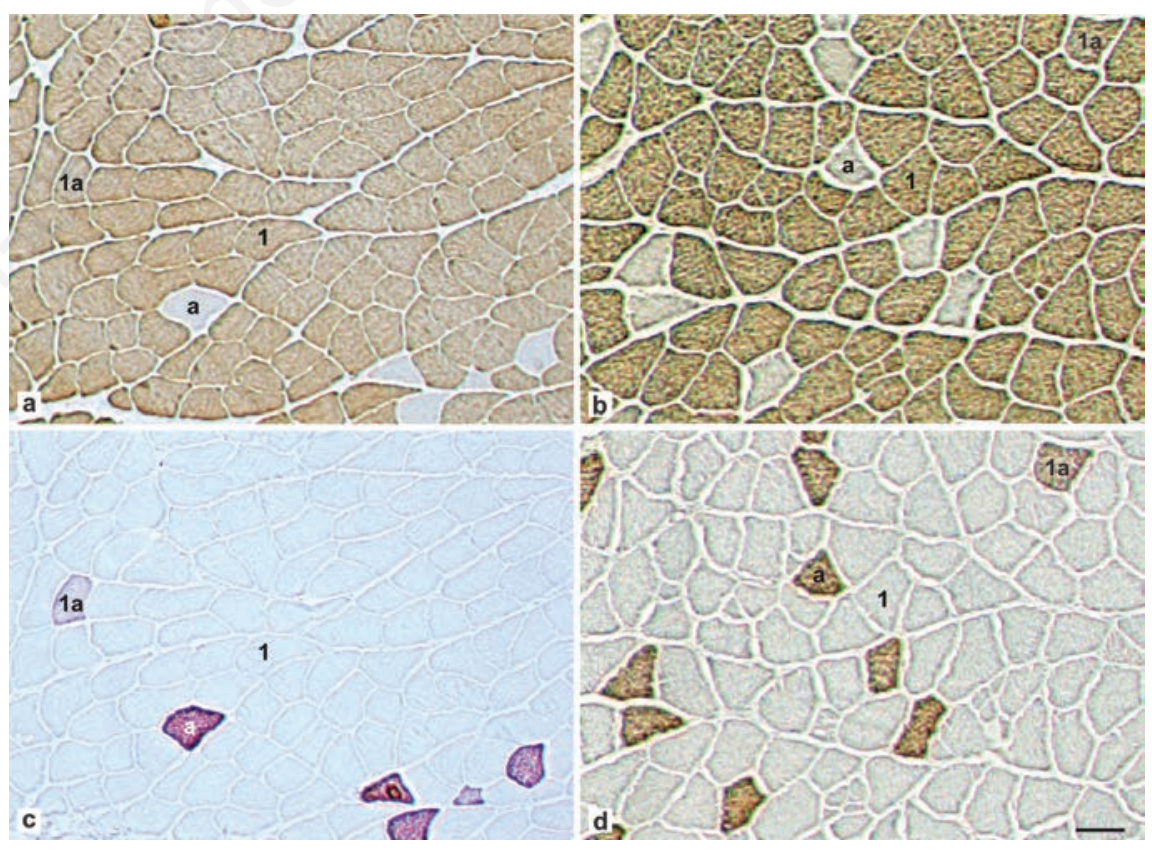

Figure 4. Effect of swimming on fibre type transitions in soleus (SOL) muscle of low-fat corn oil (LFCO) diet fed rats. Type 1 (1), 1/2a (1a) and 2a (a) fibres were immunohistochemically determined according to the myosin heavy chain (MyHC) expression with monoclonal antibodies BA-D5 specific to MyHC-1 $(a, b)$ and SC-71 specific to MyHC-2a (c,d) in LFCO fed sedentary (LFCO; a,c) and swimming rats (LFCO-S; b,d). Note that fast type $2 \mathrm{a}$ fibres are more numerous in LFCO-S (b, d). Scale bar: $100 \mu \mathrm{m}$. 
observed only some muscle-specific tendencies for fibre type transitions, i.e. increased proportion of type 1 fibres and decreased proportion of type $1 / 2 \mathrm{a}$ in SOL, increased proportion of type $1 / 2 \mathrm{a}$ in EDL and type $2 \mathrm{~b}$ in GMd. As already stated, the studies of the relationship between high fat diet and muscle fibre type transitions are scarce, especially in rats. We are familiar with only 3 studies and all of them used only healthy rats. In one study 6 -week high fat diet ( $59 \%$ of fat) reduced the proportion of type 1 fibres in SOL but increased the proportion of type 1 fibres and reduced their fibre size in EDL. ${ }^{10}$ In another study it was found that 10 weeks of high fat diet $57 \%$ of fat) resulted in an increased proportion of type 1 fibres in rat SOL, while 5 weeks of high fat diet had no effect on fibre type transitions. ${ }^{11}$ In the third study no difference in MyHC mRNA expression was found in SOL, EDL and epitrochlearis (EPI) muscles after 8 weeks of high fat diet. ${ }^{9}$ According to such controversial results, it can only be concluded that the reason for such discrepancy is likely the amount and source/composition of dietary fat in these studies. Namely, Mizunoya and co-workers fed rats with a $15 \%$ fat diet derived from different fat sources (soybean oil, n-6 PUFA-rich) vs lard (low in PUFA) vs fish oil (n-3 PUFArich) and observed that the intake of different types of dietary fats affected the fibre type composition of skeletal muscles differ- ently and in muscle-specific manner. ${ }^{12}$ In addition, our findings suggest that $\mathrm{DMH}$ induced colon tumours may be additional factor to modulate the effect of high fat diet on muscle fibre types. The underlying mechanisms involved in the skeletal muscle metabolism to adapt to high lipid availability are not known. In healthy rats, it was demonstrated that dietary lipids alter the fatty acid (FA) profile of mitochondrial membrane in multiple tissues, including skeletal muscle, which may elicit short- or long-term changes in reactive oxygen species (ROS) production by muscle mitochondria in muscle-specific manner. ${ }^{26,27}$ Namely, 8 weeks of diet with $15 \%$ fat derived from soybean oil (n-6 PUFA-rich)
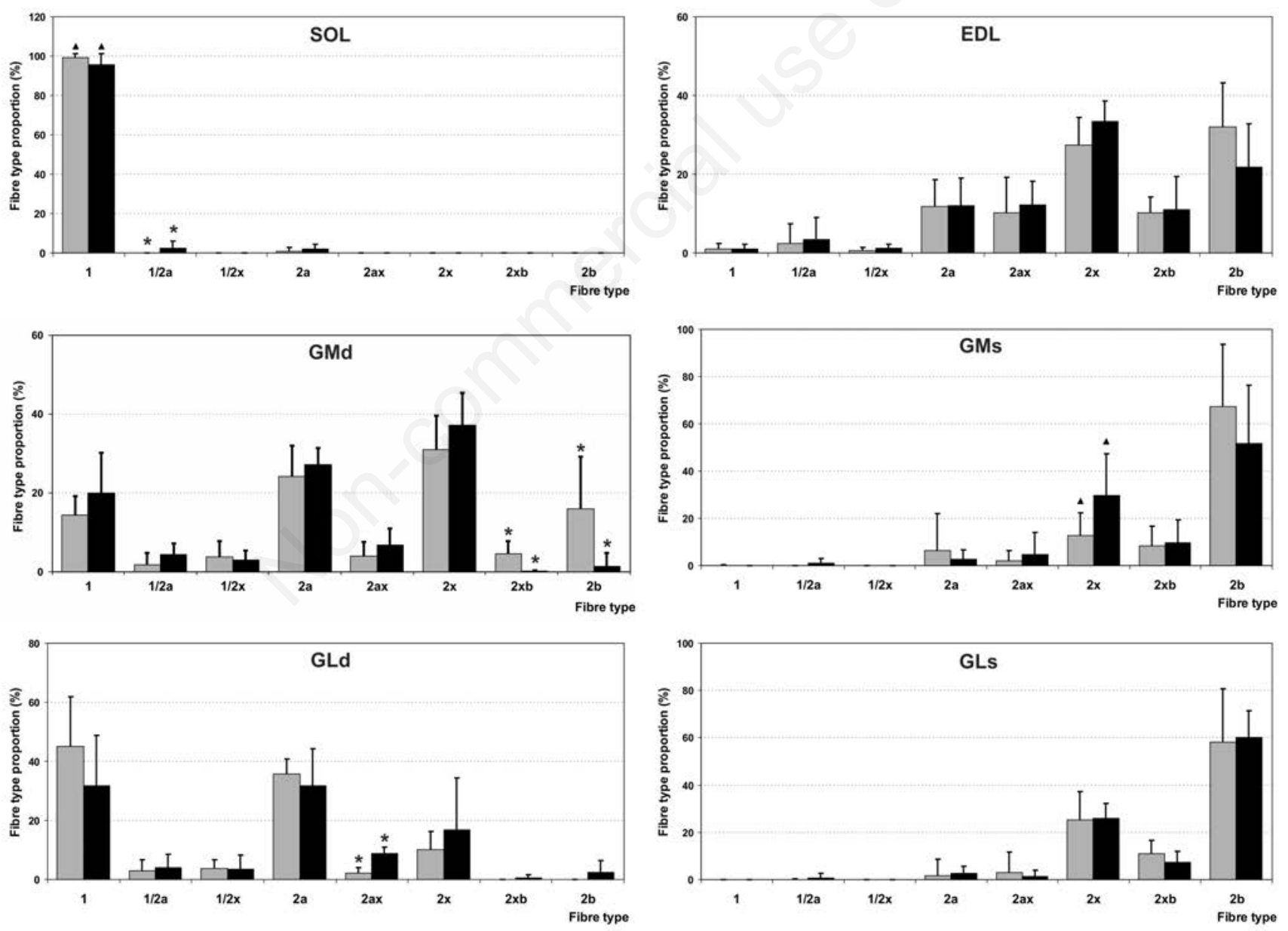

HFML $\square$ HFML-S

Figure 5. Interactive effect of high fat mixed lipid (HFML) diet and swimming on fibre type transitions: the average proportions (\%) of fibre types $(1,1 / 2 a, 1 / 2 x, 2 a, 2 a x, 2 x, 2 x b$ and $2 b)$ in the soleus (SOL), extensor digitorum longus (EDL), the deep and superficial portion of the gastrocnemius medialis (GMd, GMs) and gastrocnemius lateralis (GLd, GLs) muscles of HFML diet-fed sedentary (HFML) and swimming rats (HFML-S). Values are means \pm SEM, significant differences $(P<0.05)$ between both groups are labelled by an asterisk $(*)$ and the trends for differences $(P=0.05-0.1)$ are labelled by a triangle $(\Delta)$. 
or coconut oil (saturated FA-rich) resulted in changed activities of some antioxidant enzymes in muscle-specific manner. ${ }^{27}$ Interestingly, despite the diverse activity of ROS scavenging enzymes found in SOL and white gastrocnemius muscles after both high fat diets, an increased lipid peroxidation was found in both muscles. ${ }^{27}$ In our study in rats with DMH induced tumours 21-week, HFML diet resulted in an increased lipid peroxidation (MDA) and GSSG but with no significant differences in the activities of antioxidant enzymes (SOD, CAT, GPx, GR, GSH) in SOL muscle, indicating a compromised antioxidant defence system after HFML diet. ${ }^{18}$ It is well known that SOL muscle is rich in mitochondria and relies mostly on fat oxidation for its energy metabolism, similar like EDL muscle, containing quite high proportion of oxidative type $2 \mathrm{a}$ fibres, while EPI muscle, rich in type $2 \mathrm{~b}$ fibres, is poor in mitochondria and relies mostly on glycolytic metabolism. Thus, it is not surprising that Pinho and coworkers ${ }^{9}$ observed that the capacity of fat oxidation was similar in SOL and EDL and much lower in the EPI muscle. Although all three muscles increased their rates of fatty acid oxidation to cope with the overabundance of lipids, no significant differences in the activity of antioxidant enzymes SOD, CAT or GPx were found in these muscles after 8 weeks of high fat diet (composed of lard and soybean oil). However, in SOL significantly decreased activity of TRXT and increased UCP2 and UCP3 mRNA expression was found. ${ }^{9}$ Taken together, these results suggest that the regulation of ROS production is an intrinsic characteristic of a muscle, related to its fibre type composition (oxidative $v s$ glycolytic) and is consequently muscle-specific. Therefore, the impact of potentially deleterious effects of ROS production on skeletal muscle metabolism/ function under various conditions (high fat diet, exercise, malnutrition, tumorigenesis) also seems to be fibre-type dependent. Additionally, our studies suggest that HFML diet might importantly impair the antioxidant status of the muscles and muscle fibre type composition in rats with DMH induced colon tumours, however this observation needs further investigation.

\section{Effect of swimming}

It is well established that the endurance training induces fast-to-slow fibre type transitions in skeletal muscles. ${ }^{1,2}$ In contrast to this generally accepted paradigm, we demonstrated that in rats with $\mathrm{DMH}$ induced tumours the endurance swimming (21 weeks) induced a trend of transitions in the expected direction only in some of fast gastrocnemius samples. Interestingly, the endurance swimming resulted in increased proportion of hybrid $1 / 2 \mathrm{x}$ fibres in GMd, what is the opposite to the effect of DMH treatment in sedentary rats, ${ }^{7}$ showing that swimming has neutralized the effect of DMH treatment. To our knowledge, this is the first study demonstrating a beneficial effect of endurance swimming on muscle fibre types in rats with $\mathrm{DMH}$ induced colon tumours. Contrary to fast GMd, in slow SOL swimming induced transitions in the opposite direction (slow-to-fast). Such a diverse or muscle-specific response to swimming in healthy rats has been reported previously. Namely, in rat EDL muscle a decreased proportion of the fastest type $2 b$ and concomitant increased type $2 \mathrm{x}$ proportion were reported after 4 -week swimming indicating fast-to-slow transitions. ${ }^{28}$ Similarly, in fast plantaris muscle of Wistar rats fast-to-slow transitions were found after 8-week swimming with overload. ${ }^{29}$ On the contrary, in SOL muscle of Wistar rats slow-to-fast fibre type transitions were found after 6-week swimming ${ }^{30}$ and after 8week swimming with overload, ${ }^{29}$ which is in agreement with our results obtained after 21-week swimming in DMH-treated rats.

These results show that the direction and extent of fibre type transitions is most likely related to the functional recruitment of a muscle in a particular type of exercise. Namely, studies employing muscular blood flow and electromyography (EMG) recordings, which both reflect the recruitment level of muscles, demonstrated that SOL is recruited at swimming, but less heavily than the fast GM, though both muscles are synergists at plantar ankle flexion. ${ }^{31,32}$ Swimming is actually a non-weight bearing form of locomotion that unloads the slow postural muscle such as SOL and loads more the ankle fast-twitch extensor tibialis anterior and fast-twitch flexor gastrocnemius muscle as the activation level of GM and GL was higher during swimming than during running. ${ }^{33}$ It was also demonstrated that the involvement of GMs increases relative to GMd with increased motor demands. Contrary to swimming, during quadrupedal standing and running the highest EMG activity was recorded in SOL, whereas in GMd it was lower and GMs was silent. ${ }^{32}$ However, we assume that due to intrinsic genetically determined characteristics, the range of fibre type transitions in a muscle is related not only to the role of a particular muscle in swimming but to its basic fibre type composition as well. ${ }^{2,34}$

In addition, it was demonstrated that swimming affected the antioxidant status and mitochondrial function in skeletal muscles in muscle-specific manner as well. For instance, after 8-week swimming (60 $\mathrm{min}$ /day 5 days/week) the activity of SOD and GPx decreased, whereas that of CAT increased in white gastrocnemius muscle. While in slow SOL the activity of $\mathrm{CuZnSOD}$ and CAT remained unchanged, the activity of MnSOD increased and that of GPx decreased. The citrate synthase activity, a commonly used marker for the presence of intact mitochondria, was increased in SOL but unchanged in gastrocnemius, indicating an increase in the mitochondrial density/function in SOL after swimming. ${ }^{35}$ It is well established that ROS generated during muscle contraction play a critical role in the muscle adaptation to exerciseinduced oxidative stress by activating redox-sensitive signalling pathways (i.e., $\mathrm{NF} \kappa \mathrm{B}, \mathrm{MAPK}$ ), which govern not only the antioxidant enzyme adaptation but also other biological activities in skeletal muscle, such as mitochondrial biogenesis, repair and even fibre type transformation. ${ }^{36-}$ ${ }^{38}$ However, we found that the endurance swimming in rats with DMH induced colon tumours did not affect the antioxidant status (SOD, CAT, GPx, GR, GSH, GSSG, MDA) in SOL muscle ${ }^{16}$ although slow-to-fast fibre type transitions were induced. Our results thus indicate that the endurance swimming may abolish the effects of DMH induced colon tumours on muscle fibre types through other, currently unknown mechanisms.

\section{HFML diet compromised the effect of swimming}

To our knowledge the combined effects of HFML diet and swimming on fibre type composition of skeletal muscles of rats with $\mathrm{DMH}$ induced tumorigenesis have not been evaluated yet. We found that HFML diet not only compromised the beneficial effect of swimming on colon cancer incidence ${ }^{19}$ but on the fibre type transitions in skeletal muscles as well. Although the effect of swimming (slow-to-fast) prevailed in SOL of DMH-treated rats on HFML diet, it was partly restricted by the counteractive effect of HFML diet as the transitions proceeded only up to hybrid $1 / 2$ a fibres and not further up to type $2 \mathrm{a}$ as found in DMH-treated rats on LFCO diet. Moreover, HFML diet had almost completely neutralized the effect of swimming in SOL as the fibre type composition of SOL in HFML-S was very similar to that in LFCO fed sedentary rats. Like in SOL, HFML diet and swimming had an opposite effect in GMd as well. Namely, the tendency of type $2 b$ proportion increase due to HFML diet was obviously counteracted by swimming as the proportion of this fibre type was significantly lower in HFML-S. Thus, our results demonstrated that in rats with $\mathrm{DMH}$ induced colon tumours fibre type transitions induced by combined effects of swimming and HFML diet are muscle-specific and counteractive as well. 
Like in our study in DMH-treated rats, a muscle-specific and counteractive effects of high fat diet and exercise (running) on fibre type transitions were observed in healthy rats as well, ${ }^{10}$ which additionally confirms that high fat diet and exercise are important factors, which can trigger the fibre type transitions in various health conditions, including tumorigenesis.

The underlying mechanisms of combined effects of swimming and high fat diet involved in the metabolism/adaptation of skeletal muscles are not known but seem to affect the antioxidant status of a muscle in muscle-specific manner too. For instance, 8 weeks of diet with $15 \%$ fat derived from soybean or coconut oil resulted in altered ROS production in white gastrocnemius muscle of swimming rats while in SOL no changes in ROS production were found. Interestingly, despite the unchanged activity of antioxidant enzymes in the swimming rats, the coconut oil diet resulted in decreased activity of citrate synthase and soybean oil diet in increased lipid peroxidation in SOL muscle. ${ }^{27}$ In our previous study in rats with $\mathrm{DMH}$ induced colon tumours, 21-week swimming resulted in markedly increased ROS production in SOL muscle of rats on HFML diet, shown by a significantly increased activity of SOD, CAT, GPx, but unchanged level of MDA. ${ }^{18}$ Thus, all these results suggest that the dietary fat, depending on the composition of fatty acids, can cause various responses in ROS production under exercise conditions. In our previous study HFML diet significantly affected the antioxidant status of SOL under exercise conditions, which resulted in the muscle adaptation both at the level of antioxidant status and fibre type proportions.

Therefore, these findings show that the exercise as well as the type and amount of fat included in the diet are important factors in the muscle adaptation in rats with colon tumours. Thus, neglecting these facts can lead to the misinterpretation of the results, particularly in studies of carcinogenesis. In conclusion, we demonstrated that in rats with DMH induced colon tumours HFML diet resulted only in some trends for changes of individual fibre type proportions, whereas swimming induced more prominent fibre type transitions only in slow SOL. Interestingly, if present the effects of HFML diet and swimming on fibre type composition of skeletal muscles were counteractive. Namely, in SOL of rats with DMH induced colon tumours HFML diet had almost completely neutralized the effect of swimming. Importantly, both HFML diet and/or swimming effects were found to be muscle-specific and are probably related to the basic, genetically deter- mined muscle fibre type composition, which determines the adaptive range for fibre type transitions due to changed physiological conditions. However, further studies are necessary to elucidate the underlying mechanisms that modulate the effects of HFML diet and exercise on muscle fibre type transitions in rats with tumours.

\section{References}

1. Baldwin KM, Haddad F. Effects of different activity and inactivity paradigms on myosin heavy chain gene expression in striated muscle. J Appl Physiol (1985) 2001;90:345-57.

2. Schiaffino S, Reggiani C. Fiber types in mammalian skeletal muscles. Physiol Rev 2011;91:1447-31.

3. DeNardi C, Ausoni S, Moretti P et al. Type 2X-myosin heavy chain is coded by a muscle fiber type-specific and developmentally regulated gene. J Cell Biol 1993;123:823-35.

4. Blaauw B, Schiaffino S, Reggiani C. Mechanisms modulating skeletal muscle phenotype. Compr Physiol 2013;3: 1645-87.

5. Pette D, Staron RS. Mammalian skeletal muscle fiber type transitions. Int Rev Cytol 1997;170:143-223.

6. Booth FW, Tseng BS, Flück M, Carson JA. Molecular and cellular adaptation of muscle in response to physical training. Acta Physiol Scand 1998;162:343-50.

7. Smerdu V, Perše M. Effect of carcinogen 1,2-dimethylhydrazine treatment on fiber types in skeletal muscles of male Wistar rats. Physiol Res 2017;66:845-58.

8. Abou Mrad J, Yakubu F, Lin D, Peters JC, Atkinson JB, Hill JO. Skeletal muscle composition in dietary obesity-susceptible and dietary obesity-resistant rats. Am J Physiol 1992;262:R684-8.

9. Pinho RA, Sepa-Kishi DM, Bikopoulos $\mathrm{G}$ et al. High-fat diet induces skeletal muscle oxidative stress in a fiber typedependent manner in rats. Free Radic Biol Med 2017;110:381-9.

10. Liao B, Xu Y. Exercise improves skeletal muscle insulin resistance without reduced basal mTOR/S6K1 signaling in rats fed a high-fat diet. Eur J Appl Physiol 2011;111:2743-2752.

11. Kaneko S, Iida RH, Suga T, Fukui T, Morito M, Yamane A. Changes in triacylglycerol-accumulated fiber type, fiber type composition, and biogenesis in the mitochondria of the soleus muscle in obese rats. Anat Rec (Hoboken) 2011;294:1904-12.

12. Mizunoya W, Iwamoto Y, Shirouchi B, Sato M, Komiya Y, Razin FR, et al.
Dietary fat influences the expression of contractile and metabolic genes in rat skeletal muscle. PLoS One 2013;8 e80152.

13. Perše M. Physical activity, dietary fat and colorectal cancer. In E. Rajunor (ed) Colorectal Cancer - From prevention to patient care. Rijeka, InTech; 2012; p. 103-22.

14. Perše M, Cerar A. Morphological and molecular alterations in 1,2 dimethylhydrazine and azoxymethane induced colon carcinogenesis in rats. J Biomed Biotechnol 2011;2011:473964.

15. Perše M, Cerar A. The dimethylhydrazine induced colorectal tumours in rat - experimental colorectal carcinogenesis. Radiol Oncol 2005;39:61-70.

16. Perše M, Cerar A. The role, significance and applicability of aberrant crypt foci in clinical practice. In J. Khan J (ed) Colorectal cancer - surgery, diagnostics and treatment. Rijeka, InTech; 2014; p. 467-83.

17. Rao CV, Hirose Y, Indranie C, Reddy BS. Modulation of experimental colon tumorigenesis by types and amounts of dietary fatty acids. Cancer Res 2001;61:1927-33.

18. Perše M, Injac R, Strukelj B, Cerar A. Effects of high-fat mixed-lipid diet and exercise on the antioxidant system in skeletal and cardiac muscles of rats with colon carcinoma. Pharmacol Rep 2009;61:909-16.

19. Perše M, Injac R, Štrukelj B, Cerar A. High fat mixed lipid diet modifies protective effects of exercise on 1,2 dimethylhydrazine induced colon cancer in rats. Technol Cancer Res Treat 2012;11:289-99.

20. Matsakas A, Bozzo C, Cacciani N, Caliaro F, Reggiani C, Mascarello F, et al. Effect of swimming on myostatin expression in white and red gastrocnemius muscle and in cardiac muscle of rats. Exp Physiol 2006;91:983-94.

21. Schiaffino S, Saggin L, Viel A, Ausoni S, Sartore S, Gorza L. Muscle fibre types identified by monoclonal antibodies to myosin heavy chains. In G. Benzi, L. Packer, N. Siliprandi (ed) Biochemical aspects of physical exercise. Amsterdam: Elsevier; 1986; p. 2734.

22. Lucas CA, Kang LH, Hoh JF. Monospecific antibodies against the three mammalian fast limb myosin heavy chains. Biochem Biophys Res Commun 2000;272:303-8.

23. Gorza L. Identification of a novel type 2 fiber population in mammalian skeletal muscle by combined use of histochemical myosin ATPase and anti-myosin monoclonal antibodies. J Histochem 
Cytochem 1990;38:257-65.

24. Smerdu V, Soukup T. Demonstration of myosin heavy chain isoforms in rat and humans: the specificity of seven available monoclonal antibodies used in immunohistochemical and immunoblotting methods. Eur J Histochem 2008;52:179-90.

25. Karen P, Stevanec M, Smerdu V, Cvetko E, Kubínová L, Erzen I. Software for muscle fibre type classification and analysis. Eur J Histochem 2009;53:87-95.

26. Villalba JM, López-Domínguez JA, Chen Y, Khraiwesh H, González-Reyes JA, Del Río LF, et al. The influence of dietary fat source on liver and skeletal muscle mitochondrial modifications and lifespan changes in calorie-restricted mice. Biogerontology 2015;16:655-70.

27. Pereira B, Rosa LF, Safi DA, Guimarães AR, Bechara EJ, Curi R. Antioxidant enzyme activities in the lymphoid organs and muscles of rats fed fatty acids-rich diets subjected to prolonged physical exercise-training. Physiol Behav 1994;56:1049-55.

28. Sugiura T, Morimoto A, Sakata Y, Watanabe T, Murakami N. Myosin heavy chain isoform changes in rat diaphragm are induced by endurance training. Jpn J Physiol 1990;40:759-63.

29. Vechetti IJ, Aguiar AF, de Souza RW, Almeida FL, de Almeida Dias HB, de Aguiar Silva MA, et al. NFAT isoforms regulate muscle fiber type transition without altering $\mathrm{CaN}$ during aerobic training. Int J Sports Med 2013;34:861-7.

30. Nascimento CC, Padula N, Milani JG, Shimano AC, Martinez EZ, MattielloSverzut AC. Histomorphometric analysis of the response of rat skeletal muscle to swimming, immobilization and rehabilitation. Braz J Med Biol Res 2008;41:818-24.

31. Laughlin MH, Mohrman SJ, Armstrong RB. Muscular blood flow distribution patterns in the hindlimb of swimming rats. Am J Physiol 1984;246:H398-403.

32. Roy RR, Hutchison DL, Pierotti DJ, Hodgson JA, Edgerton VR. EMG patterns of rat ankle extensors and flexors during treadmill locomotion and swimming. J Appl Physiol (1985) 1991; 70:2522-9.

33. de Leon R, Hodgson JA, Roy RR, Edgerton VR. Extensor- and flexor-like modulation within motor pools of the rat hindlimb during treadmill locomotion and swimming. Brain Res
1994;654:241-50.

34. Soukup T, Smerdu V. Effect of altered innervation and thyroid hormones on myosin heavy chain expression and fiber type transitions: a mini-review. Histochem Cell Biol 2015;143:123-30.

35. Pereira B, Costa Rosa LF, Safi DA, Medeiros MH, Curi R, Bechara EJ. Superoxide dismutase, catalase, and glutathione peroxidase activities in muscle and lymphoid organs of sedentary and exercise-trained rats. Physiol Behav 1994; 6:1095-9.

36. Yan Z, Okutsu M, Akhtar YN, Lira VA. Regulation of exercise-induced fiber type transformation, mitochondrial biogenesis, and angiogenesis in skeletal muscle. J Appl Physiol (1985) 2011;110:264-24.

37. Merry TL, Ristow M. Mitohormesis in exercise training. Free Radic Biol Med 2016;98: 123-30.

38. Ji LL, Gomez-Cabrera MC, Vina J. Role of nuclear factor kappaB and mitogen-activated protein kinase signaling in exercise-induced antioxidant enzyme adaptation. Appl Physiol Nutr Metab 2007;32:930-5. 